\title{
Central Pontine Myelinolysis (CPM): An Atypical Presentation of HIV
}

\author{
Kushwaha Suman ${ }^{\mathrm{a}, \mathrm{d}}$, Malhotra Hardeep ${ }^{\mathrm{b}}$, Garg Sushil ${ }^{\mathrm{a}}$, Maheshwari Siddharth ${ }^{\mathrm{a}}$, \\ Malik Seema ${ }^{a}$, Goyal Renu ${ }^{\mathrm{c}}$
}

\begin{abstract}
HIV affects the Nervous System at different stages of disease ranging from central nervous system to peripheral nervous system. Nervous system manifestation of HIV is estimated to affect approximately $25-90 \%$ of the patients .In spite of wide range of the manifestations of neurological complications Central Pontine Myelinolysis (CPM) remains an uncommon presentation of HIV. A sixty year male presented with history of sub acute onset of fever, quadriparesis and dysarthria of two month duration. This case discusses the atypical neurological presentation in HIV patient.
\end{abstract}

Keywords: HIV; Atypical presentation; Neurological manifestation

\section{Introduction}

A wide range of nervous system involvement occurs in HIV [1].Central Pontine Myelinolysis is an uncommon condition

Manuscript accepted for publication April 6, 2012

${ }^{\mathrm{a}}$ Department of Neurology, Institute of Human Behaviour and Allied Sciences, Delhi, India

${ }^{\mathrm{b}}$ Department of Neurology, C.S.M Medical University, Lucknow, India

${ }^{\mathrm{c}}$ Department of Microbiology, Institute of Human Behaviour and Allied Sciences, Delhi, India

${ }^{\mathrm{d}}$ Corresponding author: Kushwaha Suman.

Email: sumankushwaha@gmail.com

doi:10.4021/jnr92w characterized by the clinical Syndrome of quadriparesis, pseudo bulbar palsy and symmetrical lesions in the center of basis pontis. The risk factors associated with CPM includes presence of hypoxemia, preexisting hypokelemia, malnutrition and chronic alcoholism. It is frequently associated with rapid correction of hyponatremia [2]. The clinical presentation varies between rapidly evolving spastic paraparesis with pseudo bulbar palsy, and changes in sensorium with confusion or coma. Some cases are clinically 'silent' and are only discovered at autopsy. CPM in patients with HIV infection is usually not recognized in life. There are few reports of CPM occurring in HIV patients with identified risk factors for developing CPM [3]. Diagnosing a case presenting with signs and symptoms of CPM in HIV patient with no identified risk factor is difficult.

\section{Case Report}

A 60 years old male presented with history of low grade fever of 2 month duration along with sub acute onset of quadriparesis and slurring of speech. He had altered sensorium of 1 week duration. The patient was admitted in neurology ICU. On Physical examination he was average built. His vitals were stable pulse rate $86 / \mathrm{min}$ regular, BP: $130 / 70 \mathrm{mmHg}$, R/R 16/min, Afebrile. Cardiovascular, respiratory system and per abdomen examination was not significant. On Neurological examination: Patient was in altered sensorium, obeying commands with gestures and by making incomprehensible sounds. Cranial nerves - Fundus - Normal. He had bilateral lateral gaze paresis. Pupils were normal size reacting to light. Gag reflex was brisk . Rest other cranial nerves were normal. The muscle power was $3 / 5$ on MRC grading in all the limbs. All the Deep tendon reflexes were exaggerated. Planters were bilaterally withdrawal.

\section{Laboratory investigations}

Hb: $10 \mathrm{~g} \%$, TLC: 5700 cumm, Differential counts was normal. Random Blood $-153 \mathrm{mg} / \mathrm{dL}$, renal function test were normal. ESR: $77 \mathrm{~mm}$ 1st hr. Serum electrolytes- S. $\mathrm{Na}^{+}: 138$ meq/L, Serum Sodium remained normal throughout hospital 


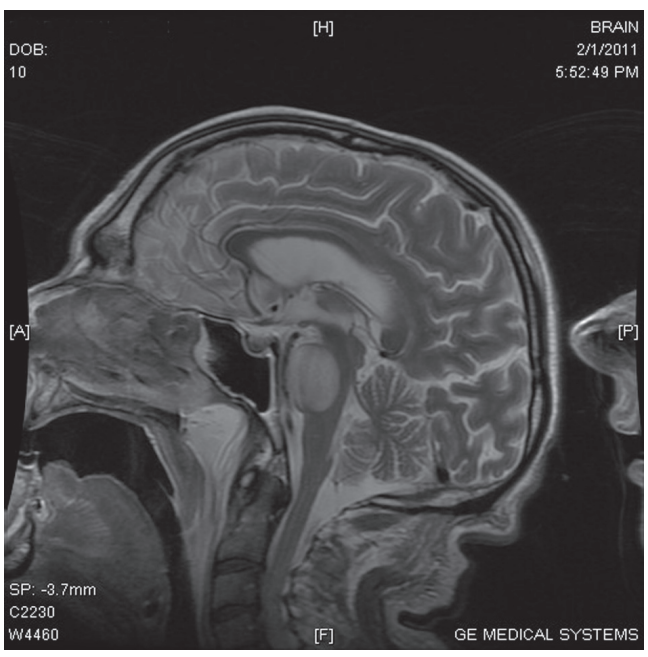

Figure 1. MRI Brain - Mid saggital section shows A well demarcated hyperintensity in pons.

stay. Liver function test-Se Bilirubin $0.8 \mathrm{mg} / \mathrm{dL}$, SGOT 24 U/L, SGPT 28 U/L, APL 183 U/L, Serum cholesterol -182 mg/dL. Serum Proteins - 7.1 g/dL, Albumin 3.6 g/dL, Serum Osmolality $285 \mathrm{mmol} / \mathrm{dL}$, CSF Examination - Cytology Acellular, Sugar: $54 \mathrm{mg} / \mathrm{dl}$, Protein $79 \mathrm{mg} / \mathrm{dL}$, Chloride 113 $\mathrm{mmol} / \mathrm{L}$. All the biochemical parameters were within normal limits. CD4 count was $250 / \mathrm{mL}$. HIV I and II antibodies were detected by using three different methods e.g. Elisa/ Rapid and simple test in patients serum.

He was started on antiretroviral therapy and symptomatic treatment.

\section{MRI Brain}

Bilateral Symmetrical Hyper intense signals are seen in pons consistent with central pontine Myelinolysis (Fig. 1, 2).

\section{Discussion}

Central pontine Myelinolysis (CPM) was first described in 1959 in four patients, three of whom were alcoholic and one was severely malnourished. This clinicopathological syndrome is characterized by quadriparesis, pseudo bulbar palsy and symmetric lesion in centre of basis pontis. Extrapontine Myelinolysis also occurs and patient can have varied presentation according to the site involved. The unusual clinical presentations include ataxia, catatonia, neurobehavioral Syndromes and extra pyramidal involvement. CPM is usually found in sick patients with malnutrition, malignancy, burn injury, chronic alcoholism, hypoxemia and pre-existing hypokelemia. HIV associated CPM had been documented in Autopsy by various authors [4]. There are few reports of CPM occurring in AIDS patients with potential risk factors

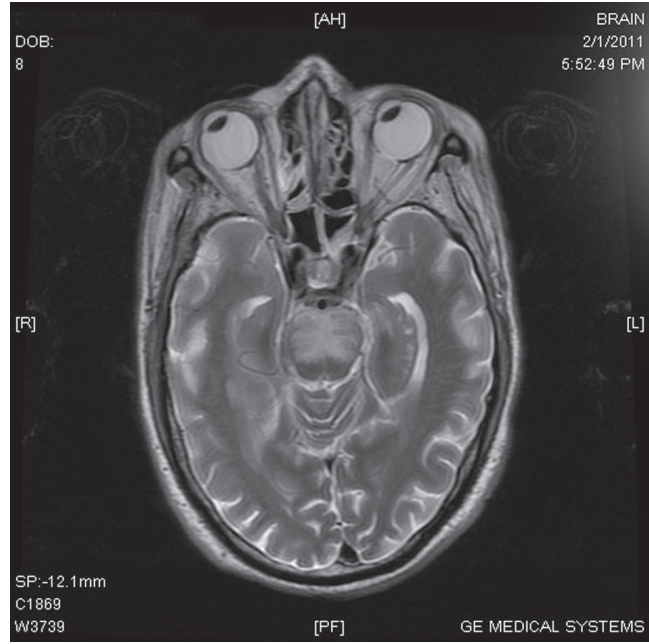

Figure 2. MRI Brain - HIV produces Symmetrical Demyelination of Pons. Axial T2 weighted images -The lesion in Pons shows typical Bats Wing or Trident appearance.

for CPM development [5]. There has been no previous report of CPM presenting in as neurological manifestation in HIV patient. Our case is unique as CPM is documented without any risk or precipitating factor. There was no evidence of electrolytic imbalance in our patient. His albumin levels were normal. CPM has been described in liver transplant recipients and in severe burns cases, as well as in association with alcoholism and malnutrition. It has been thought that the rapid correction, or over correction, of hyponatremia was the crucial factor in causing CPM in most studied cases. Our patient did not have an episode of serum hyperosmolality or hypoalbuminaemia. The Diagnosis was made on clinical examination which was consistent with CPM and the neuroimaging confirmed the involvement of pons (Fig. 1). In absence of the risk factors or precipitating factors the possibility is that CPM may be thought as a primary effect of HIV itself, representing another neurological manifestation of this virus which is not well known and needs to be studied. The p24 HIV protein was detected immunocytochemically in rare perivascular macrophages in the brain stem and beyond the margin of the area involved with CPM in neuropath logical studies. Further studies are required to understand the effect of HIV on the demyelinization of pons and other brain structures.

\section{Conclusion}

CPM is demyelinization syndrome of the pons. In HIV patients it is usually not recognized during life and diagnosed on autopsy. Clinical presentation may vary, ranging from mutism and dysarthia to spastic quadriparesis with a pseudo bulbar palsy. Our case illustrates that CPM should be considered as the cause for neurological manifestation in patients with AIDS in the appropriate clinical setting, regardless of 
presence or absence of the risk factors responsible for causing the fatal condition.

\section{References}

1. McArthur JC, Brew BJ, Nath A. Neurological complications of HIV infection. Lancet Neurol. 2005;4(9):543555.

2. Tormey WP. Central pontine myelinolysis and changes in serum sodium. Lancet. 1990;335(8698):1169.

3. Apoola A, Ross J, Duddy MJ, Mudaliar V, Jones EL,
Huengsberg M, Miller RF. Central pontine myelinolysis complicating treatment of multicentric Castleman's disease and Kaposi's sarcoma in a patient with AIDS. Sex Transm Infect. 2003;79(3):179-184.

4. Kure K, Llena JF, Lyman WD, Soeiro R, Weidenheim KM, Hirano A, Dickson DW. Human immunodeficiency virus-1 infection of the nervous system: an autopsy study of 268 adult, pediatric, and fetal brains. Hum Pathol. 1991;22(7):700-710.

5. Holmes AH, Esiri M, Morris CS, Edwards A. Central pontine myelinolysis in a patient with AIDS. J Neurol Neurosurg Psychiatry. 1992;55(7):631-632. 